\title{
Identification of a second $\beta$-glucoside phosphoenolpyruvate : carbohydrate phosphotransferase system in Corynebacterium glutamicum R
}

Correspondence

Hideaki Yukawa

mmg-lab@rite.or.jp

Received 25 March 2009

Revised 17 July 2009

Accepted 20 July 2009
Yuya Tanaka, Haruhiko Teramoto, Masayuki Inui and Hideaki Yukawa

Research Institute of Innovative Technology for the Earth, 9-2, Kizugawadai, Kizugawa, Kyoto 619-0292, Japan

The phosphoenolpyruvate : carbohydrate phosphotransferase system (PTS) catalyses carbohydrate transport by coupling it to phosphorylation. Previously, we reported a Corynebacterium glutamicum R $\beta$-glucoside PTS encoded by bgIF. Here we report that $C$. glutamicum $\mathrm{R}$ contains an additional $\beta$-glucoside PTS gene, bgIF2, organized in a cluster with a putative phospho- $\beta$-glucosidase gene, bgIA2, and a putative antiterminator, $b g / G 2$. While single gene disruption strains of either bgIF or bgIF2 were able to utilize salicin or arbutin as sole carbon sources, a double disruption strain exhibited defects in utilization of both carbon sources.

Expression of both $b g / F$ and $b g / F 2$ was induced in the presence of either salicin or arbutin, although disruption of $b g / G 2$ affected only bg/F2 expression. Moreover, in the presence of either salicin or arbutin, glucose completely repressed the expression of bgIF but only slightly repressed that of bgIF2. We conclude that BgIF and BglF2 have a redundant role in $\beta$-glucoside transport even though the catabolite repression control of their encoding genes is different. We also show that expression of both $b g / F$ and $b g I F 2$ requires the general PTS.

\section{INTRODUCTION}

The phosphoenolpyruvate: carbohydrate phosphotransferase system (PTS) catalyses transport of carbohydrates by coupling carbohydrate translocation to phosphorylation (Postma et al., 1993; Kotrba et al., 2001a). The PTS consists of two common cytoplasmic proteins, enzyme I (EI) and $\mathrm{HPr}$, and an array of sugar-specific enzyme II complexes (EIIs). The phosphoryl group from phosphoenolpyruvate is sequentially transferred to EI, HPr, EIIs and finally to the substrate as it is translocated across the membrane.

The PTS also has a regulatory role in response to carbohydrate availability. In the presence of a rapidly metabolizable carbon source such as glucose, bacteria often preferentially utilize this carbon source by repressing expression of other catabolic genes. This phenomenon is called carbon catabolite repression. Phosphorylation states of PTS components have a critical role in carbon catabolite repression (Postma et al., 1993; Deutscher et al., 2006). In Escherichia coli, phosphorylation of EIIA ${ }^{\text {Glc }}$ regulates the level of cyclic AMP, which binds to CRP protein, a

Abbreviations: PRD, PTS regulation domain; PTS, phosphoenolpyruvate : carbohydrate phosphotransferase system; RAT, ribonucleic antiterminator.

A supplementary table of primers is available with the online version of this paper. transcriptional regulator of many catabolic genes. In addition, unphosphorylated EIIA ${ }^{\text {Glc }}$ inhibits many sugar transporters, resulting in the decrease of inducers in the cell (inducer exclusion). In Bacillus subtilis and other low-GC Gram-positive bacteria, HPr binds to CcpA protein to control the transcription of many catabolic genes. HPr is also involved in inducer exclusion in low-GC Grampositive bacteria (Postma et al., 1993; Deutscher et al., 2006).

Expression of the $\beta$-glucoside PTS in many bacteria is upregulated in response to the substrate, and is often subject to strict glucose repression. In E. coli, B. subtilis and many other bacteria, expression of the $\beta$-glucoside PTS gene is controlled by an antitermination mechanism. In antitermination regulation, transcription terminates at a terminator present in the 5' UTR of mRNA in the absence of substrate. In the presence of substrate, BglG/SacY family transcriptional antiterminators recognize the ribonucleic antiterminator (RAT) sequence present in the 5' UTR of mRNA (Stülke \& Hillen, 2000; Amster-Choder, 2005; Deutscher et al., 2006). RAT partially overlaps the transcriptional terminator, and it is believed that binding of transcriptional antiterminator to RAT stabilizes the secondary structure of the RAT sequence and disrupts the terminator structure, which results in the elongation of transcripts to the end of the target gene. The BglG/SacY 
family antiterminator contains the PTS regulation domain (PRD). Phosphorylation of the PRD-1 site by EII of the PTS inhibits antiterminator activity, which is the underlying mechanism of upregulation of the $\beta$-glucoside PTS gene in response to the substrate. On the other hand, PRD2 site phosphorylation by HPr is required for antitermination, which is related to glucose repression.

Corynebacterium glutamicum is a non-pathogenic high-GC Gram-positive soil bacterium which is widely used for the industrial production of amino acids, notably glutamic acid and lysine (Kinoshita et al., 1957; Kelle et al., 2005). C. glutamicum $\mathrm{R}$ can provide high yields of lactate and succinate from sugar (Inui et al., 2004a; Okino et al., 2008a, b). In C. glutamicum, the general PTS (EI and HPr) as well as glucose-, fructose- and sucrose-specific PTS EIIs (Mori \& Shiio, 1987; Dominguez \& Lindley, 1996; Kotrba et al., 2001b; Parche et al., 2001; Moon et al., 2005), and additional $p t s$ genes $\left(c g R \_2922\right.$ and $c g R \_2923$ of $C$. glutamicum R, cg3365 and cg3366 of C. glutamicum ATCC 13032) for unidentified substrates are present. However, a full understanding of the regulatory role of the PTS in C. glutamicum remains elusive. C. glutamicum can simultaneously utilize multiple carbon sources, and preferential utilization of glucose has been reported in only a few cases (Dominguez et al., 1997; Wendisch et al., 2000). The global catabolite repression mechanism involving the key roles of the PTS, as found in E. coli and B. subtilis, has not been identified in C. glutamicum.

A distinct feature of C. glutamicum $\mathrm{R}$ is that, unlike $C$. glutamicum strains ATCC 13032 and ATCC 13869, it can utilize the $\beta$-glucosides salicin and arbutin, which are transported via a $\beta$-glucoside-specific PTS (Kotrba et al., 2003). The $\beta$-glucoside phosphotransferase and utilization system in C. glutamicum R comprises three genes, bglF (cgR_2729), bglA (cgR_2728) and $b g l G\left(c g R \_2727\right)$, encoding $\beta$-glucoside PTS (EIIBCA ${ }^{\mathrm{bgl}}$ ), phospho- $\beta$-glucosidase and positive transcription regulator, respectively. Efficient expression of the $\beta$-glucoside PTS requires the $b g l G$ gene. BglG shows $53 \%$ similarity to LicT from $B$. subtilis and BglG from E. coli, two transcriptional antiterminators belonging to the $\mathrm{BglG} / \mathrm{SacY}$ family. A RAT-like sequence and a putative transcriptional terminator are present upstream of the $C$. glutamicum $\mathrm{R}$ bglF gene, suggesting that C. glutamicum $\mathrm{R}$ $b g l F$ is regulated via the antitermination mechanism.

A C. glutamicum $\mathrm{R}$ strain with a deleted $b g l F$ gene still consumes $\beta$-glucosides (Kotrba et al., 2003), suggesting that this strain encodes another $\beta$-glucoside transporter. In this study, we describe identification of a new $\beta$-glucoside phosphotransferase and utilization system in C. glutamicum R. It comprises three genes, bglF2 (cgR_2610), bglA2 (cgR_2609) and bglG2 (cgR_2608), putatively encoding EIIBCA $^{\text {bgl }}$, phospho- $\beta$-glucosidase and antiterminator, respectively. We also investigate involvement of antiterminators and general PTS components in the regulation of $b g l F$ and $b g l F 2$ in response to the presence of $\beta$-glucoside and glucose.

\section{METHODS}

Media and growth conditions. C. glutamicum $\mathrm{R}$ was grown aerobically at $33{ }^{\circ} \mathrm{C}$ with shaking at 200 r.p.m. For the analysis of sugar concentration, C. glutamicum $\mathrm{R}$ was grown in $100 \mathrm{ml} \mathrm{A}$ medium (Inui et al., 2007) supplemented with $1 \%$ sugar, or in BT minimal medium (Inui et al., 2007) supplemented with $0.2 \%$ sugar. For the analysis of $b g l$ expression, C. glutamicum $\mathrm{R}$ was grown in $10 \mathrm{ml}$ A medium supplemented with $1 \%(\mathrm{w} / \mathrm{v})$ carbon sources. Bacterial growth was monitored by determining the $\mathrm{OD}_{610}$.

Bacterial strains and plasmids. The strains used in this study are as follows. C. glutamicum R (Yukawa et al., 2007) was used as a wildtype strain. The strains with various gene deletions ( $b g l F, b g l F 2, b g l G$, bglG2, ptsI and $p t s H$ ) were constructed as described previously (Inui et al., 2004b). A suicide vector, pCRA725, carrying the $s a c B$ gene was used for construction of markerless gene deletions. Oligonucleotide primers used for gene deletion are summarized in Supplementary Table S1, available with the online version of this paper. Briefly, DNA fragments encoding genes with an internal deletion were cloned into pCRA725. The resultant plasmids were introduced into C. glutamicum $\mathrm{R}$ and single-crossover cells were isolated by using kanamycin resistance. Isolated cells were cultivated on BT medium supplemented with $10 \%$ sucrose and double-crossover cells were isolated. The gene deletions were confirmed by DNA sequencing of the PCR products around the deleted region.

The strains having bglF- or bglF2-lacZ fusion genes (PbglF-lacZ or $\mathrm{P} b g l F 2-l a c Z$ ) were constructed as described previously (Tanaka et al., 2008a). The promoter region of $b g l F$ or $b g l F 2$ was amplified by PCR using primers EcoRV-bglF1-400-F and EcoRV-bglF1-9-R for the $b g l F$ promoter and EcoRV-bglF2-400-F and EcoRV-bglF2-9-R for the bglF2 promoter. The amplified fragment was digested with $E c o R V$ and cloned into the DraI site of the pCRA741 reporter plasmid, which is described elsewhere (Inui et al., 2007). Construction of mutants deleted for both RAT and the transcriptional terminator region of the bglF- or bglF2lacZ fusion genes (PbglFARAT-lacZ or PbglF2 $\triangle R A T-l a c Z$ ) was conducted as follows. The plasmids containing bglF- or bglF2-lac $Z$ were used as a template for inverse PCR using primer sets RI-bglF1$120-\mathrm{R}$ and RI-bglF1-60-F for the bglF promoter and RI-bglF2-150-R and RI-bglF2-60-F for the $b g l F 2$ promoter. The amplified fragment was digested with EcoRI and self-ligated. These RAT-transcriptional terminator deletion plasmids were used to transform C. glutamicum $\mathrm{R}$ and recombinant cells were selected for kanamycin resistance. Insertion of the promoter-lacZ fusion gene between cgR_0734 and $c g R \_0735$ was confirmed by PCR using primers LlacZLR-4354F and Ind7insert-checkR, or LlacZLR-6425R and Ind7insert-checkF.

Analysis of sugars. C. glutamicum cells were centrifuged $(1000 \mathrm{~g}$, $4{ }^{\circ} \mathrm{C}, 1 \mathrm{~min}$ ), and supernatants were analysed for sugars. The concentration of salicin was measured by high-performance liquid chromatography (8020, Tosoh) with a $600 \mathrm{~mm}$ OA PAK-A TSK-GEL column (Tosoh). Sugars were detected with a refraction index detector.

Real-time RT-PCR. Total RNA was isolated from exponentially growing cells $\left(\mathrm{OD}_{610} 1.2\right)$ using the RNeasy kit (Qiagen). A $20 \mathrm{ng}$ sample of total RNA was used as template for analysis of the $b g l$ genes and $0.4 \mathrm{ng}$ was used for analysis of the 16S rRNA to generate cDNA and for the subsequent PCR. Each real-time RT-PCR mixture $(20 \mu \mathrm{l})$ contained $500 \mathrm{nM}$ of each primer, $10 \mu \mathrm{l}$ of Power SYBR Green PCR Master Mix, eight units of RNase Inhibitor and five units of MuLV reverse transcriptase (Applied Biosystems). The primers used in these reactions are listed in Supplementary Table S1. Reactions were performed using the ABI 7500 Fast Real-Time PCR System (Applied Biosystems) with the following cycle parameters: one cycle of $50{ }^{\circ} \mathrm{C}$ for $30 \mathrm{~min}$ and $95{ }^{\circ} \mathrm{C}$ for $10 \mathrm{~min}$, followed by 40 cycles of $95{ }^{\circ} \mathrm{C}$ for $15 \mathrm{~s}$ and $60{ }^{\circ} \mathrm{C}$ for $30 \mathrm{~s}$. The result of $16 \mathrm{~S}$ rRNA was used as an internal control. 


\section{RESULTS}

\section{Identification of a second set of $\beta$-glucoside- utilization genes}

In a previous paper, we described the presence of a $\beta$ glucoside PTS encoded by bglF in C. glutamicum R (Kotrba et al., 2003). However, disruption of the $b g l F$ gene did not prevent consumption of $\beta$-glucosides (Kotrba et al., 2003). We inferred that there is another $\beta$-glucoside-utilizing system in C. glutamicum R. A search of the entire C. glutamicum R genome (Yukawa et al., 2007) for candidate $\beta$-glucoside utilization genes revealed CgR_2610, CgR_2609 and CgR_2608, with $38 \%$ amino acid sequence identity and $57 \%$ similarity to BglF, $40 \%$ identity and $56 \%$ similarity to BglA, and $34 \%$ identity and $53 \%$ similarity to BglG, respectively. We designated $c g R \_2610$, $c g R \_2609$ and $c g R \_2608$ as bglF2, bglA2 and $b g l G 2$, respectively (Fig. 1a). We compared the amino acid sequence of BglF2 with BglF (Fig. 1b). We also compared it with that of PtsG because BglF is a member of the PtsG family of EII (Postma et al., 1993). Comparison of BglF2, $\mathrm{BglF}$ and PtsG revealed that the regions around the putative phosphorylation site on the EIIB domain (Cys30 residue of $\mathrm{BglF} 2$ ) and the EIIA domain (His-575 residue of BglF2) are highly conserved (Fig. 1b). We previously reported that point mutation on the EIIC domain

(a)

(b)
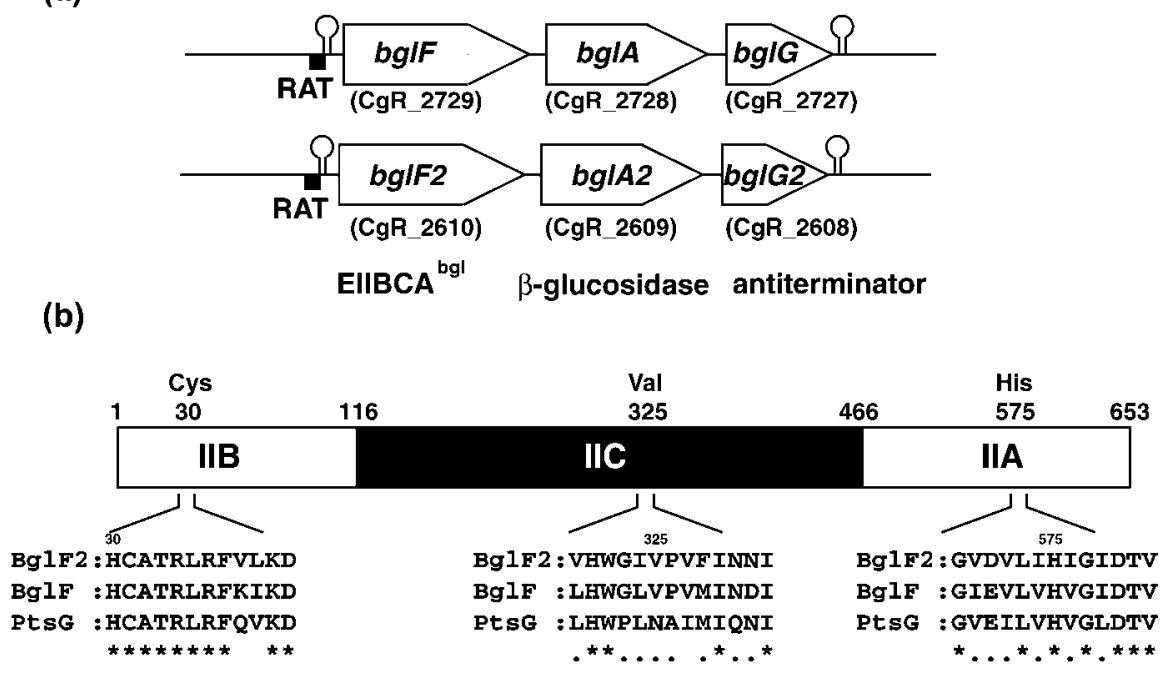

(c)

TTTTCTTGTCTGGGGCGGAGTGGGTTCGAAACACTCTTGCATTTTTCGG

AAGAATTGATCTAGAGTAAATTTCAGTTACATAAGCAACAGTCCATCAGG GTTGTTATTCCAGTCACTGGAAGCAAAACCIGGTCACCAAATGCTGAATG

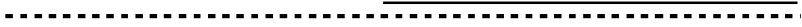
TGTCTCGTTTTAGGATGCATTTTCGCCACCGGTGGCCAGGTTTTTTGCAT

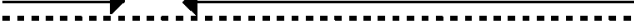

TTCAGAAACGGATGGAAAGCAGTCCTTAAAGGTTTTTAGGGAGGTCCATCATG (d)

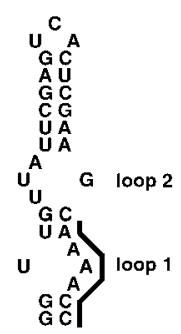

Fig. 1. Two $\beta$-glucoside utilization systems in C. glutamicum R. (a) Organization of the two bg/ gene clusters. Open arrows represent the coding regions for indicated genes. Deduced functions of each gene are indicated below. RAT-like sequences and putative transcriptional terminators are indicated as boxes and stem-loops, respectively. (b) Comparison of amino acid sequence between BgIF, BglF2 and PtsG. The region around the phosphorylation site of the EllB domain (amino acid residues 29-40 of BglF2), the substrate specifity domain of EIIC (residues 305-333 of BgIF2) and the phosphorylation site of EllA domain (residues 569-581 of BglF2) are shown. Conserved residues in two of three Ells are indicated by dots, and conserved residues in all three Ells are indicated by asterisks. The putative phosphorylation site in BglF2 (Cys-30 and His-575) and Val-325, in which mutation in BgIF results in cellobiose recognition, are indicated. (c) Sequence of the upstream region of bgIF2. The sequence of the region upstream of the translation initiation codon is shown. The region which has similarity with the RAT sequence is boxed. The palindromic sequence which partially overlaps with the RAT-like sequence is indicated by a pair of inverted arrows. The dashed line indicates the deleted region in the PbgIF2 $2 R A T$-lacZ strain. (d) Secondary structure of the RAT-like sequence of bgIF2. The line to the right of the hairpin indicates the $5^{\prime}$ region of the palindromic sequence. The secondary structure shown is based on comparison with the proposed secondary structure of other RAT sequences (Yang et al., 2002). 
(permease domain) of EIIBCA $^{\text {bgl }}$ (V317A or V317M) allowed the cells to transport cellobiose, which is not a substrate for wild-type EIIBCA ${ }^{\text {bgl }}$, suggesting that this amino acid is important for substrate recognition. Val-317 of $\mathrm{BglF}$ is conserved in BglF2 but not conserved in PtsG.

\section{Involvement of BgIF and BgIF2 in $\beta$-glucoside utilization}

We tested the growth of a strain with deleted $b g l F 2$ on a $\beta$ glucoside (salicin) as the sole carbon source (Fig. 2a). A disruptant lacking either $b g l F$ or $b g l F 2$ grew on minimal medium with salicin as well as did the wild-type. Simultaneous disruption of both $b g l F$ and $b g l F 2$ resulted in severe defects in growth on salicin. We also tested the growth on plates supplemented with several $\beta$-glucosides (Table 1). Wild-type C. glutamicum $\mathrm{R}$ grows on arbutin, methyl $\beta$-D-glucoside and salicin, but not on cellobiose (Kotrba et al., 2003). A disruptant lacking a general component of the PTS ( $p t s H$ or $p t s I$ ) did not grow on any of the tested $\beta$-glucosides, suggesting that these sugars are transported by the PTS or, alternatively, expression and/or activities of $\beta$-glucoside sugar utilization system are controlled by the PTS. Single disruption of either bglF or bglF2 did not affect the utilization of any of the carbon sources tested. Simultaneous disruption of $b g l F$ and $b g l F 2$ resulted in defective growth on arbutin and salicin, while colony formation was observed on methyl $\beta$-D-glucoside. These results indicate that both BglF and BglF2 transport arbutin and salicin. It is likely that another transport system is involved in methyl $\beta$-D-glucoside transport, although we did not rule out that BglF and/or BglF2 are also involved in the transport.

To test whether $b g l F 2$ is involved in $\beta$-glucoside utilization, sugar concentration in the medium was measured in aerobic growth conditions (Fig. $2 \mathrm{~b}, \mathrm{c}$ ). Because the $b g l F$ bglF2 double disruptant does not grow on minimal medium with salicin, we used nutrient-rich medium (A medium) supplemented with $0.2 \%$ salicin or arbutin (Fig. $2 \mathrm{~b}, \mathrm{c}$ ). Wild-type, $b g l F$ disruptant, and $b g l F 2$ disruptant strains showed similar growth while the bglF bglF2 double disruptant strain showed slower growth and lower final cell density (data not shown). Salicin and arbutin concentrations decreased almost by equal measure during the growth of wild-type, $b g l F$ mutant and $b g l F 2$ mutant strains, and the $\beta$-glucosides were completely utilized in $8 \mathrm{~h}$ cultivation. The bglF bglF2 double disruptant showed very slow consumption of salicin or arbutin. Therefore, we concluded that both BglF and BglF2 are able to transport salicin and arbutin, and also postulate that both are major $\beta$-glucoside transporters.

\section{Regulation of bgIF2 expression by $\boldsymbol{\beta}$-glucosides}

Expression of the $b g l F$ and $b g l F 2$ genes was investigated by RT-PCR analysis (Fig. 3). Expression of many bgl genes in other bacteria is subject to glucose repression. Therefore, (a)

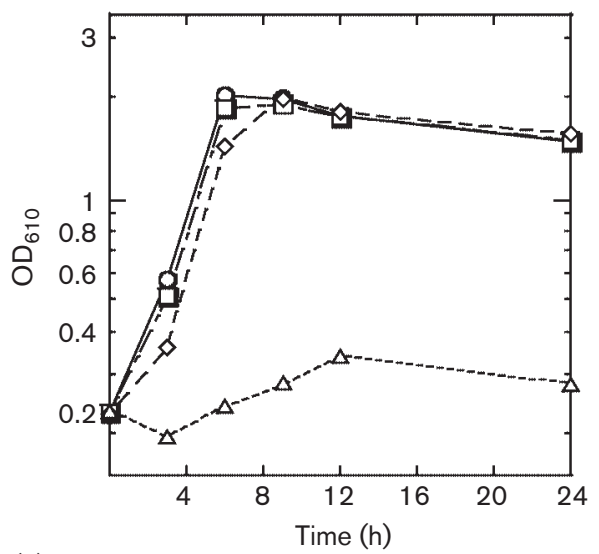

(b)

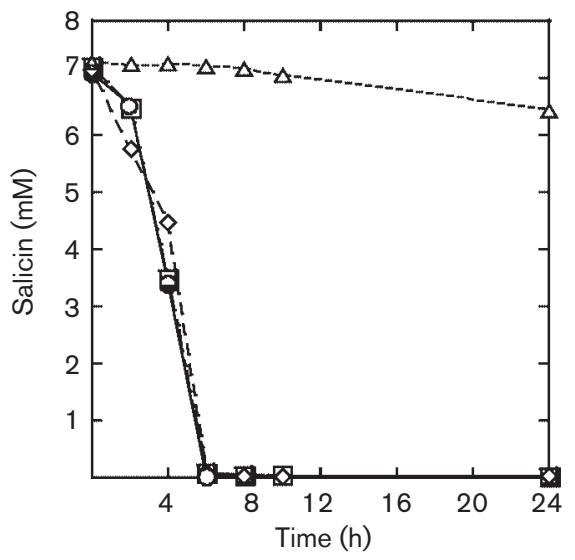

(c)

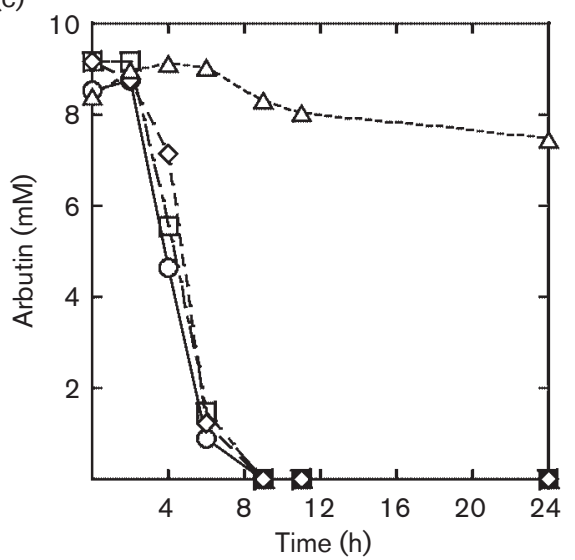

Fig. 2. $\beta$-Glucoside utilization by $b g /$ mutant strains. (a) Growth of wild-type and $\mathrm{bg} /$ mutants in minimal medium with $0.2 \%$ salicin. (b, c) Concentration of salicin (b) and arbutin (c) in the culture medium of wild-type and $\mathrm{bg} /$ mutants grown in A medium with $0.2 \%$ salicin or $0.2 \%$ arbutin, respectively. $\bigcirc$, Wild-type; $\square$, bglF; $\diamond$, bglF2; $\triangle$, bgIF bgIF2. The results shown are representative of at least two independent reproducible experiments.

we tested the effects of glucose added in the presence of salicin (Fig. 3a, b) or arbutin (Fig. 3c, d). We also tested the effect of fructose because expression of general as well as 
Table 1. Utilization of $\beta$-glucosides by $b g /$ gene deletion strains

Minimal BT medium plates were supplemented with $1 \%(\mathrm{w} / \mathrm{v})$ carbon sources as indicated. Growth on plates visible after $24 \mathrm{~h}$ is indicated as + .

\begin{tabular}{|lcccccc|}
\hline & WT & ptsH & ptsI & bglF & bglF2 & bglF \\
& & & & & & bglF2 \\
\hline Arbutin & + & - & - & + & + & - \\
Methyl $\beta$-D-glucoside & + & - & - & + & + & + \\
Salicin & + & - & - & + & + & - \\
Cellobiose & - & - & - & - & - & - \\
\hline
\end{tabular}

sugar-specific pts genes is strongly induced in the presence of fructose. The levels of $b g l F$ and $b g l F 2$ mRNAs did not increase upon the addition of glucose or fructose. This indicates that the regulatory mechanism of $b g l F$ and $b g l F 2$ is different from that of $p t s$ genes that are controlled by the transcriptional repressor protein SugR (Engels \& Wendish, 2007; Gaigalat et al., 2007; Tanaka et al., 2008b). Indeed, we found that $b g l F$ and $b g l F 2$ mRNA levels did not increase upon the disruption of $s u g R$ gene (data not shown). As expected, $b g l F$ and $b g l F 2$ expression was increased in the presence of salicin (Fig. 3a, b). The level of $b g l F$ mRNA was repressed to less than $10 \%$ by the addition of glucose (Fig. 3a, column 5). Addition of fructose partially repressed the expression of $b g l F$ (Fig. 3a, column 6). In contrast, expression of $b g l F 2$ was decreased by only $20 \%$ by the addition of glucose (Fig. 3b, column 5) and by $50 \%$ by addition of fructose (Fig. 3b, column 6). Similar results were obtained with arbutin (Fig. 3c, d). These results indicate that $b g l F$ and $b g l F 2$ are under different catabolite repression control by glucose.

\section{Regulation of bgIF2 expression by BgIG2 independent of BgIG}

We found that $b g l G 2$, coding for an antiterminator-like protein, is located near the bglF2 gene on the $C$. glutamicum $\mathrm{R}$ chromosome. Moreover, there is a RATlike sequence which overlaps a palindromic sequence (Fig. 1c, d) upstream of $b g l F 2$. These observations suggest that (a)

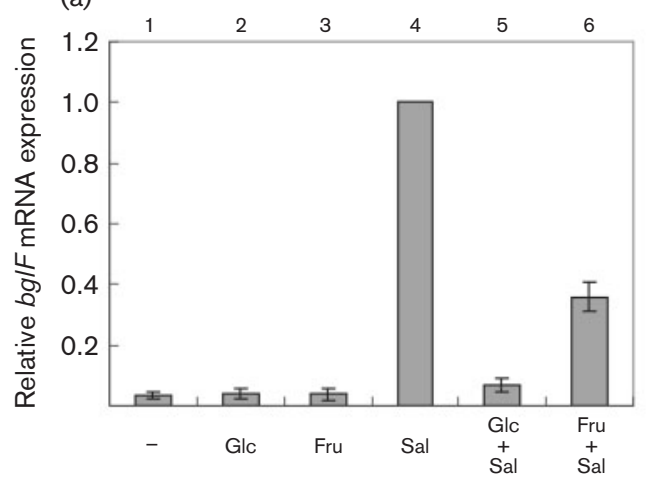

(c)

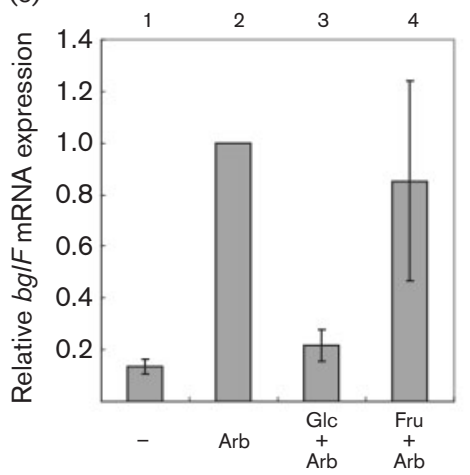

(b)

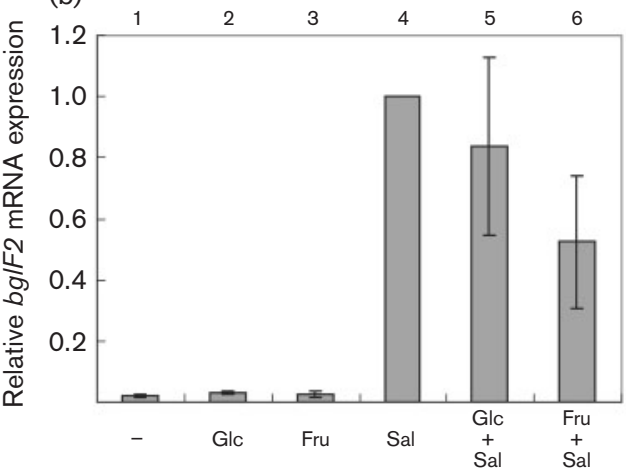

(d)

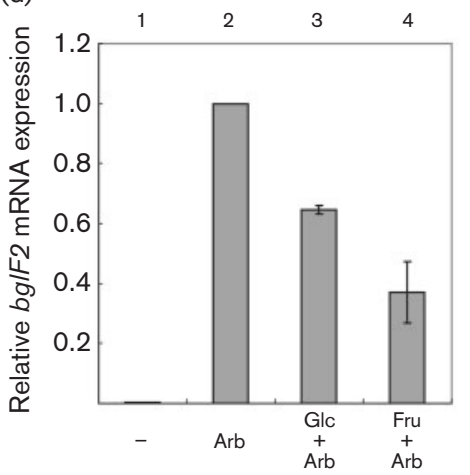

Fig. 3. Expression of $\mathrm{bg} /$ genes in the presence of various PTS sugars. Total RNAs prepared from wild-type cells grown in A medium supplemented or not with $1.0 \%(\mathrm{w} / \mathrm{v})$ PTS sugars were subjected to real-time RT-PCR analysis using primers specific for $\operatorname{bg} I F(\mathrm{a}, \mathrm{c})$ or bgIF2 $(\mathrm{b}, \mathrm{d})$. The relative mRNA level is indicated as the ratio compared to cells grown in the presence of salicin. The values are the means of three independent experiments and standard deviations are indicated. Abbreviations: Glc, glucose; Fru, fructose; Sal, salicin; Arb, arbutin. 
bglF2 expression is controlled by an antitermination mechanism via BglG2. To confirm this, a strain with deleted $b g l G 2$ was constructed and bglF2 expression determined by RT-PCR analysis. As described previously (Kotrba et al., 2003), bglF induction by salicin was not observed in the $b g l G$ mutant strain. The level of $b g l F$ mRNA was increased upon addition of salicin in the bglG2 mutant strain, suggesting that BglG2 is not involved in the regulation of $b g l F$ expression (Fig. 4a). Expression of $b g l F 2$ was not affected by $b g l G$ deletion (Fig. 4b). Deletion of bglG2 resulted in a marked decrease in salicin induction of bglF2 (Fig. 4b). The slight induction of $b g l F 2$ by salicin in the $b g l G 2$ disruption strain was completely abolished in a bglG bglG2 double disruptant strain. These results indicate that $\mathrm{BglG2}$ regulates the expression of $b g l F 2$, while there may be only minor cross-talk regulation by BglG of $b g l F 2$ expression.

To further test whether or not antitermination is involved in the induction of $b g l F$ and $b g l F 2$ genes, we examined effects of deletion of the putative RAT sequence and transcriptional terminator sequence on the promoter-lac $Z$ gene fusions that integrated into the chromosome of $C$. glutamicum R. The levels of lacZ mRNA were determined by RT-PCR. As expected, the expression levels of the bglFand bglF2-lacZ fusions were very low in the absence of

(a)

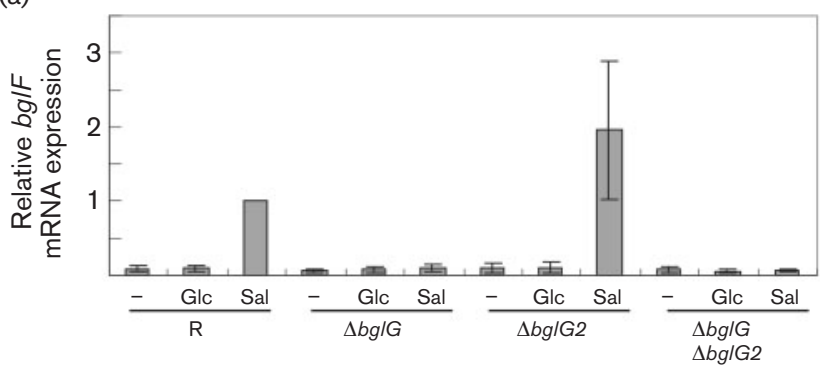

(b)

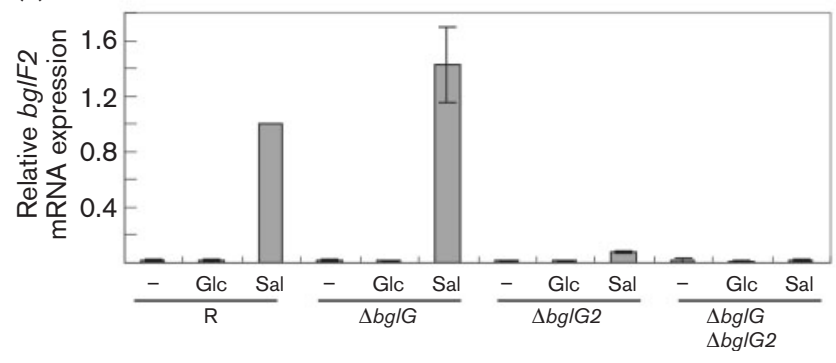

Fig. 4. Effect of $b g / G$ or $b g / G 2$ disruption on $b g / F$ and $b g / F 2$ gene expression. Total RNAs prepared from cells grown in A medium supplemented or not with $1.0 \%$ (w/v) PTS sugars were subjected to real-time RT-PCR analysis using primers specific for $b g I F(a)$ or bgIF2 (b). The relative mRNA level is indicated as the ratio compared to cells grown in the presence of salicin. The values are the means of three independent experiments and standard deviations are indicated. Abbreviations: Glc, glucose; Fru, fructose; Sal, salicin. salicin, but markedly induced in its presence (the relative lac $Z$ expression levels in the absence of salicin were $0.042 \pm 0.023$ and $0.070 \pm 0.054$ for PbglF- and PbglF2lac $Z$, respectively, compared to those in the presence of salicin). Deletion of the putative cis-element for antitermination resulted in the expression of lac $Z$ even in the absence of salicin, and expression was barely induced by salicin (the relative lac $Z$ expression levels in the absence of salicin were $0.81 \pm 0.08$ and $1.48 \pm 0.20$ for PbglFARATlacZ and PbglF2 $\Delta R A T-l a c Z$, respectively, compared to those in the presence of salicin). These results indicate that the RAT-termination region is essential for the regulation of $b g l F$ and $b g l F 2$.

\section{Effect of PTS disruption on bgl expression}

The PTS is generally involved in the regulation of the activity of BglG/SacY-type transcriptional antiterminators. To test whether the PTS is involved in the regulation of the activity of BglG and/or BglG2, we investigated $b g l F$ and bglF2 gene expression in ptsI (encoding EI) or $p t s H$ (encoding $\mathrm{HPr}$ ) mutants by RT-PCR analysis. In the absence of salicin, the levels of $b g l F$ and $b g l F 2$ mRNAs were equally low in the wild-type, ptsI mutant and $p t s H$ mutant strains (Fig. 5a, b). The marked induction of $b g l F$ and $b g l F 2$ by supplementation with salicin observed in the wild-type strain was completely suppressed in the ptsI or pts $\mathrm{H}$ mutant strains (Fig. 5a, b). These results indicate that the PTS is

(a)

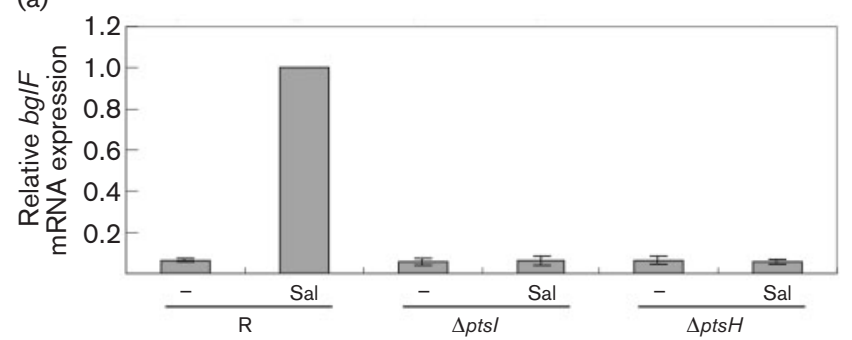

(b)

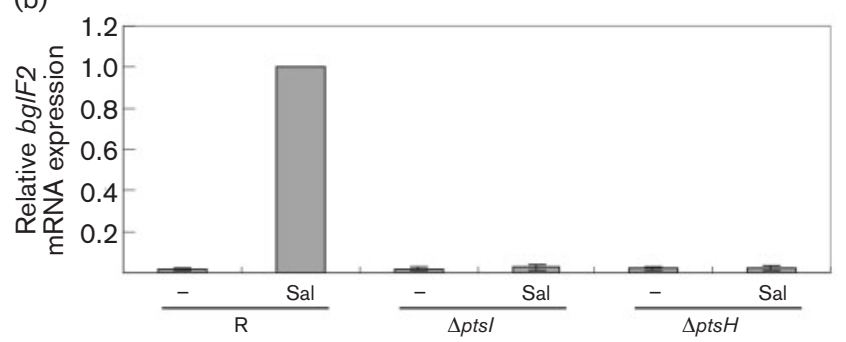

Fig. 5. Effect of disruption of the general PTS on bg/F and bg/F2 expression. Total RNAs prepared from cells grown in A medium supplemented or not with $1.0 \%(\mathrm{w} / \mathrm{v})$ salicin (Sal) were subjected to real-time RT-PCR analysis using primers specific for $b g I F(a)$ or bgIF2 (b). The relative mRNA level is indicated as the ratio compared to cells grown in the presence of salicin. The values are the means of three independent experiments and standard deviations are indicated. 
required for the induction of $b g l F$ and $b g l F 2$ expression in response to salicin.

\section{DISCUSSION}

In this paper, we report a second $\beta$-glucoside PTS encoded by $\operatorname{bglF2}$, which is organized on the $C$. glutamicum $\mathrm{R}$ chromosome in a cluster with a phospho- $\beta$-glucosidase gene, $b g l A 2$, and an antiterminator-like gene, bglG2. The gene organization is similar to that of the previously identified $\beta$-glucoside utilization system ( $b g l F-b g l A-b g l G$ ) of this strain. Although a $b g l F 2$ mutant grew on $\beta$-glucoside as the sole carbon source, as does a bglF mutant, simultaneous disruption of $b g l F$ and $b g l F 2$ abolished utilization of $\beta$-glucosides, and expression of these genes was similarly induced in the presence of $\beta$-glucoside. These results indicate that these two $\beta$-glucoside PTSs in $C$. glutamicum $\mathrm{R}$ are redundantly responsible for $\beta$-glucoside uptake.

Unlike C. glutamicum R, C. glutamicum ATCC 13032 does not grow on $\beta$-glucosides as sole carbon source. However, comparison of the genome sequence of $C$. glutamicum strains R (Yukawa et al., 2007) and ATCC 13032 (Ikeda \& Nakagawa, 2003; Kalinowski et al., 2003) revealed that fragments of the genes corresponding to $b g l A-b g l G$ and bglF2 do exist in C. glutamicum ATCC 13032 (Fig. 6). Protein Cg2995 predicted from the C. glutamicum ATCC 13032 genome showed $96 \%$ sequence identity to part of BglF2 of C. glutamicum R (amino acid residues 105-155), and Cg2996 also showed $94 \%$ identity to another part of BglF2 (residues 157-226). Cg2994 showed $97 \%$ amino acid sequence identity to CgR_2607 of C. glutamicum R, and Cg2999 showed $99 \%$ identity to CgR_2611, indicating that bglF2 and the cg2995-2996 region are present in the same position in the genome of the respective strains. BLAST searches revealed no region corresponding to bglA2 or bglG2 in the C. glutamicum ATCC 13032 genome, suggesting that these genes have been lost in this strain. Similarly, ORFs corresponding to fragments of $b g l G$ and bglA were found in the C. glutamicum ATCC 13032 genome (cg3144, cg3146 and cg3147), but no sequence in this strain was found to correspond to $b g l F$ of $C$. glutamicum R. An ORF encoding a 175 aa product which overlaps $b g l G$ and $b g l A$ but inversely directed can be assigned in both these strains. We have not tested whether or not this gene is functional. These findings suggest that both the bglF-bglA-bglG and bglF2-bglA2-bglG2 gene clusters were present in the common ancestor of $C$. glutamicum R and ATCC 13032. Then, both of these gene clusters were lost in C. glutamicum ATCC 13032 after the divergence into these strains. Differences in the environmental nutritional conditions that these strains have been subjected to may have led to the genetical alterations to the sugar utilization systems.

In this study, we demonstrated that expression of bglF2 requires BglG2, which belongs to the BglG/SacY-type antiterminator family (Fig. 4a, b). The RAT-like sequence found upstream of $b g l F 2$ is probably recognized by BglG2, leading to upregulation of $b g l F 2$ expression by antitermination. Indeed, we found that in the absence of the RAT-transcriptional terminator region, lacZ reporter genes from $b g l F$ and $b g l F 2$ were constitutively expressed. The

(a)

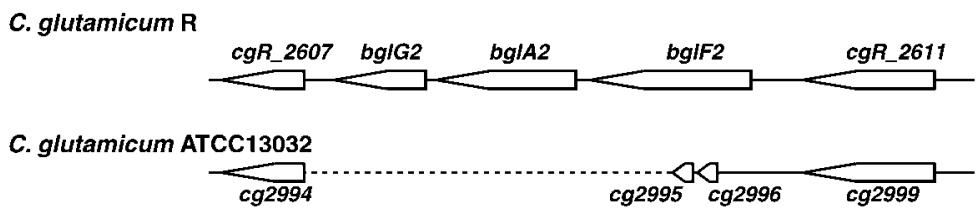

(b)

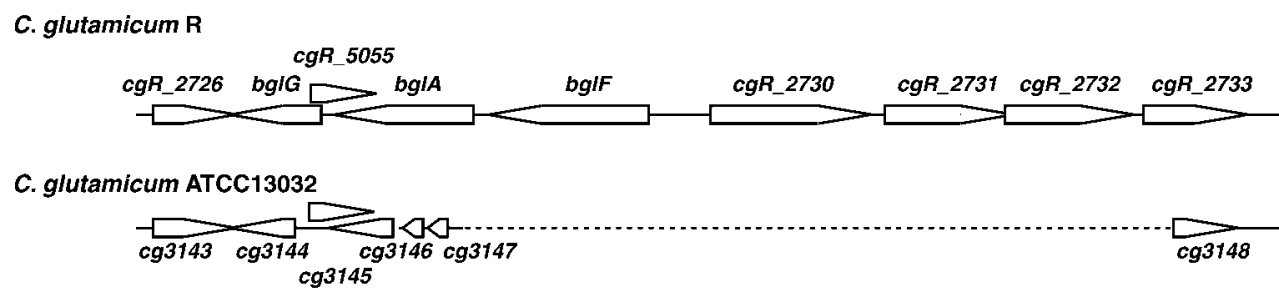

Fig. 6. Schematic representation of the organization of the genes around bg/F2 (a) and bg/F (b) in C. glutamicum R and C. glutamicum ATCC 13032. Open arrows represents the ORFs. Deduced functions of the proteins endoded by the genes are as follows: CgR_2607, putative secreted or membrane protein; CgR_2611, putative ferredoxin reductase; CgR_2726, putative secreted protein; CgR_5055, hypothetical protein; CgR_2730, ferrienterobactin-binding periplasmic protein precursor FepB2; CgR_2731, ferric enterobactin transport protein; CgR_2732, putative iron-siderophore uptake system transmembrane protein; CgR_2733, ferric enterobactin transport system ATP-binding protein FepC). The dashed line indicates the deletion in C. glutamicum ATCC 13032. 
$\mathrm{BglG} / \mathrm{SacY}$ family antiterminator is known to be involved in PTS-dependent induction and carbon catabolite repression of catabolic genes in bacteria. As with $E$. coli and $B$. subtilis $\beta$-glucoside utilization genes, it is possible that $C$. glutamicum $\mathrm{R} \beta$-glucoside PTS EII, BglF and/or BglF2 phosphorylate the PRD-1 site conserved in BglG/SacY-type antiterminators, BglG and/or BglG2, to inhibit the activity of the antiterminators, and expression of $b g l F$ and $b g l F 2$ is induced by activation of the antiterminators dephosphorylated at the PRD-1 site along with uptake and phosphorylation of $\beta$-glucoside by the substrate-specific PTS EIIs. Our results revealed that there is only minor cross-talk regulation by $\mathrm{BglG}$ of $b g l F 2$ and by $\mathrm{BglG} 2$ of $b g l F$.

Interestingly, expression of $b g l F 2$ was not repressed in the presence of glucose plus salicin, in contrast to $b g l F$ expression, which is subject to strict glucose repression. The difference in glucose repression may be due to distinct features of the antiterminators, BglG and BglG2. It is known that phosphorylation of several BglG/SacY-type antiterminators at the PRD-2 site by HPr is required for activation, and its dephosphorylation due to a decreased level of phosphorylated HPr in the presence of readily metabolizable carbon sources such as glucose could explain catabolite repression (Postma et al., 1993; Deutscher et al., 2006). It is noteworthy that activity of $B$. subtilis SacY and GlcT, belonging to the BglG/SacY-type antiterminators, whose target genes are not subject to glucose repression, does not depend on HPr. This suggests the possibility that $C$. glutamicum R BglG2 may not be under the control of the HPr-dependent PRD-2 phosphorylation. However, this possibility is ruled out by analysis of $b g l F 2$ gene expression using mutants of a general PTS component, EI or HPr (Fig. 5). If antitermination is regulated by both PRD-1 and PRD-2 phosphorylation, $p t s I$ or $p t s H$ mutation would result in the inactivation of antiterminator, while if antitermination is regulated by PRD-1 and not PRD-2, ptsI or $p t s H$ mutation would result in the activation of antiterminator. We revealed that in ptsI mutant and $p t s H$ mutant cells, expression of $b g l F$ and $b g l F 2$ was repressed in the presence and absence of salicin; this behaviour is different from that of B. subtilis SacY- and GlcT-regulated genes, whose expression is upregulated in $p t s H$ mutant cells. It should be noted that addition of fructose partially repressed induction levels of both $b g l F$ and $b g l F 2$ in the presence of salicin, which may be explained by decrease of PRD-2 phosphorylation of BglG and BglG2. To assess HPr-dependent regulation, it is important to determine the phosphorylation level of HPr in the presence of various PTS sugars.

The strong effect of glucose but not fructose on $b g l F$ expression in C. glutamicum $\mathrm{R}$ may be due to an unidentified catabolite repression mechanism other than antitermination. In B. subtilis, transcription initiation is also the target of CcpA-mediated catabolite repression of bglPH (Krüger \& Hecker, 1995). One possible mechanism to explain the effect of glucose in C. glutamicum $\mathrm{R}$ is that a transcriptional regulator that responds to the presence of glucose is involved in the regulation of $b g l F$ expression but not in the regulation of $b g l F 2$ expression. Further studies are needed to elucidate the regulatory system underlying the different behaviour of $b g l F$ and $b g l F 2$ in response to glucose but not fructose.

The presence of redundant $\beta$-glucoside PTSs is found in other bacteria, such as E. coli, Streptococcus mutans and Pectobacterium carotovorum (Parker \& Hall, 1988; Cote et al., 2000; Hong et al., 2006). However, there are few studies on how the expression of these $\beta$-glucoside PTSs is coordinated. The two $\beta$-glucoside utilization systems in C. glutamicum $\mathrm{R}$ under different catabolite repression control shown in this study may serve to fine tune the expression level. It is possible that in the presence of $\beta$ glucoside and glucose, the requirement for $\beta$-glucoside transporter is not high, so only BglF2 is induced. In the absence of glucose on the other hand, the requirement for $\beta$-glucoside transporter increases and both BglF and BglF2 are induced.

We have developed C. glutamicum R mutants of the $\beta$ glucoside PTS that can utilize cellobiose in industrial applications (Kotrba et al., 2003; Sasaki et al., 2008). In addition, the expression pattern of the $b g l F 2$ transcription system indicates that this promoter and regulator system may serve as a suitable inducible promoter, because its expression is efficiently repressed in the absence of inducer and strongly induced by the addition of inducer even in the presence of glucose.

\section{ACKNOWLEDGEMENTS}

We thank C. A. Omumasaba (RITE) for critical reading of the manuscript. This work was partially supported by a grant from the New Energy and Industrial Technology Development Organization (NEDO).

\section{REFERENCES}

Amster-Choder, O. (2005). The $b g l$ sensory system: a transmembrane signaling pathway controlling transcriptional antitermination. Curr Opin Microbiol 8, 127-134.

Cote, C. K., Cvitkovitch, D., Bleiweis, A. S. \& Honeyman, A. L. (2000). A novel beta-glucoside-specific PTS locus from Streptococcus mutans that is not inhibited by glucose. Microbiology 146, 1555-1563.

Deutscher, J., Francke, C. \& Postma, P. W. (2006). How phosphotransferase system-related protein phosphorylation regulates carbohydrate metabolism in bacteria. Microbiol Mol Biol Rev 70, 9391031.

Dominguez, H. \& Lindley, N. D. (1996). Complete sucrose metabolism requires fructose phosphotransferase activity in Corynebacterium glutamicum to ensure phosphorylation of liberated fructose. Appl Environ Microbiol 62, 3878-3880.

Dominguez, H., Cocaign-Bousquet, M. \& Lindley, N. D. (1997). Simultaneous consumption of glucose and fructose from sugar mixtures during batch growth of Corynebacterium glutamicum. Appl Microbiol Biotechnol 47, 600-603.

Engels, V. \& Wendish, V. H. (2007). The DeoR-type regulator SugR represses expression of $p t s G$ in Corynebacterium glutamicum. J Bacteriol 189, 2955-2966. 
Gaigalat, L., Schlüter, J. P., Hartmann, M., Mormann, S., Tauch, A., Pühler, A. \& Kalinowski, J. (2007). The DeoR-type transcriptional regulator SugR acts as a repressor for genes encoding the phosphoenolpyruvate:sugar phosphotransferase system (PTS) in Corynebacterium glutamicum. BMC Mol Biol 8, 104.

Hong, S. Y., An, C. L., Cho, K. M., Lee, S. M., Kim, Y. H., Kim, M. K., Cho, S. J., Lim, Y. P., Kim, H. \& Yun, H. D. (2006). Cloning and comparison of third beta-glucoside utilization ( $\operatorname{cglEFIA}$ ) operon with two operons of Pectobacterium carotovorum subsp. carotovorum LY34. Biosci Biotechnol Biochem 70, 798-807.

Ikeda, M. \& Nakagawa, S. (2003). The Corynebacterium glutamicum genome: features and impacts on biotechnological processes. Appl Microbiol Biotechnol 62, 99-109.

Inui, M., Murakami, S., Okino, S., Kawaguchi, H., Vertès, A. A. \& Yukawa, H. (2004a). Metabolic analysis of Corynebacterium glutamicum during lactate and succinate productions under oxygen deprivation conditions. J Mol Microbiol Biotechnol 7, 182-196.

Inui, M., Kawaguchi, H., Murakami, S., Vertès, A. A. \& Yukawa, H. (2004b). Metabolic engineering of Corynebacterium glutamicum for fuel production under oxygen deprivation conditions. J Mol Microbiol Biotechnol 8, 243-254.

Inui, M., Suda, M., Okino, S., Nonaka, H., Puskás, L. G., Vertès, A. A. \& Yukawa, H. (2007). Transcriptional profiling of Corynebacterium glutamicum metabolism during organic acid production under oxygen deprivation conditions. Microbiology 153, 2491-2504.

Kalinowski, J., Bathe, B., Bartels, D., Bischoff, N., Bott, M., Burkovski, A., Dusch, N., Eggeling, L., Eikmanns, B. J. \& other authors (2003). The complete Corynebacterium glutamicum ATCC 13032 genome sequence and its impact on the production of L-aspartate-derived amino acids and vitamins. J Biotechnol 104, 5-25.

Kelle, R., Hermann, T. \& Bathe, B. (2005). L-Lysine production. In Handbook of Corynebacterium glutamicum, pp. 465-488. Edited by L. Eggeling \& M. Bott. Boca Raton, FL: CRC Press.

Kinoshita, S., Udaka, S. \& Shimono, M. (2004). Studies on the amino acid fermentation. I. Production of L-glutamic acid by various microorganisms. J Gen Appl Microbiol 50, 331-343.

Kotrba, P., Inui, M. \& Yukawa, H. (2001a). Bacterial phosphotransferase system (PTS) in carbohydrate uptake and control of carbon metabolism. J Biosci Bioeng 92, 502-517.

Kotrba, P., Inui, M. \& Yukawa, H. (2001b). The ptsI gene encoding enzyme I of the phosphotransferase system of Corynebacterium glutamicum. Biochem Biophys Res Commun 289, 1307-1313.

Kotrba, P., Inui, M. \& Yukawa, H. (2003). A single V317A or V317M substitution in Enzyme II of a newly identified beta-glucoside phosphotransferase and utilization system of Corynebacterium glutamicum R extends its specificity towards cellobiose. Microbiology 149, 1569-1580.

Krüger, S. \& Hecker, M. (1995). Regulation of the putative $b g l P H$ operon for aryl-beta-glucoside utilization in Bacillus subtilis. J Bacteriol 177, 5590-5597.

Moon, M. W., Kim, H. J., Oh, T. K., Shin, C. S., Lee, J. S., Kim, S. J. \& Lee, J. K. (2005). Analyses of enzyme II gene mutants for sugar transport and heterologous expression of fructokinase gene in Corynebacterium glutamicum ATCC 13032. FEMS Microbiol Lett 244, 259-266.

Mori, M. \& Shiio, I. (1987). Phosphoenolpyruvate, sugar phosphotransferase systems and sugar metabolism in Brevibacterium flavum. Agric Biol Chem 51, 2671-2678.

Okino, S., Noburyu, R., Suda, M., Jojima, T., Inui, M. \& Yukawa, H. (2008a). An efficient succinic acid production process in a metabolically engineered Corynebacterium glutamicum strain. Appl Microbiol Biotechnol 81, 459-464.

Okino, S., Suda, M., Fujikura, K., Inui, M. \& Yukawa, H. (2008b). Production of D-lactic acid by Corynebacterium glutamicum under oxygen deprivation. Appl Microbiol Biotechnol 78, 449-454.

Parche, S., Burkovski, A., Sprenger, G. A., Weil, B., Krämer, R. \& Titgemeyer, F. (2001). Corynebacterium glutamicum, a dissection of the PTS. J Mol Microbiol Biotechnol 3, 423-428.

Parker, L. L. \& Hall, B. G. (1988). A fourth Escherichia coli gene system with the potential to evolve $\beta$-glucoside utilization. Genetics 119, 485-490.

Postma, P. W., Lengeler, J. W. \& Jacobson, G. R. (1993). Phosphoenolpyruvate, carbohydrate phosphotransferase systems of bacteria. Microbiol Rev 57, 543-594.

Sasaki, M., Jojima, T., Inui, M. \& Yukawa, H. (2008). Simultaneous utilization of D-cellobiose, D-glucose, and D-xylose by recombinant Corynebacterium glutamicum under oxygen-deprived conditions. Appl Microbiol Biotechnol 81, 691-699.

Stülke, J. \& Hillen, W. (2000). Regulation of carbon catabolism in Bacillus species. Annu Rev Microbiol 54, 849-880.

Tanaka, Y., Teramoto, H., Inui, M. \& Yukawa, H. (2008a). Regulation of expression of phosphoenolpyruvate: carbohydrate phosphotransferase system (PTS) genes in Corynebacterium glutamicum. Microbiology 154, 264-274.

Tanaka, Y., Teramoto, H., Inui, M. \& Yukawa, H. (2008b). Regulation of expression of general components of the phosphoenolpyruvate: carbohydrate phosphotransferase system (PTS) by the global regulator SugR in Corynebacterium glutamicum. Appl Microbiol Biotechnol 78, 309-318.

Wendisch, V. F., de Graaf, A. A., Sahm, H. \& Eikmanns, B. J. (2000). Quantitative determination of metabolic fluxes during coutilization of two carbon sources, comparative analyses with Corynebacterium glutamicum during growth on acetate and/or glucose. J Bacteriol 182, 3088-3096.

Yang, Y., Declerck, N., Manival, X., Aymerich, S. \& Kochoyan, M. (2002). Solution structure of the LicT-RNA antitermination complex: CAT clamping RAT. EMBO J 21, 1987-1997.

Yukawa, H., Omumasaba, C. A., Nonaka, H., Kos, P., Okai, N., Suzuki, N., Suda, M., Tsuge, Y., Watanabe, J. \& other authors (2007). Comparative analysis of the Corynebacterium glutamicum group and complete genome sequence of strain R. Microbiology 153, 1042-1058.

Edited by: C. W. Chen 